\title{
The "Journal of Functional Morphology and Kinesiology" Journal Club Series: Highlights on Recent Papers in Articular Cartilage Tissue Engineering and Mechanical Stimulation
}

\author{
Marta Anna Szychlinska ${ }^{1, *}$, Gianluca Vadalà ${ }^{2}$, Victoria Workman ${ }^{3}$, Ugo Ripamonti ${ }^{4}$, \\ Alexandrina Ferreira Mendes ${ }^{5}$, Martin J. Stoddart ${ }^{6}$ and Mauro Alini ${ }^{6}$ \\ 1 Department of Biomedical and Biotechnological Sciences, Human Anatomy and Histology Section, \\ School of Medicine, University of Catania, 95123 Catania, Italy \\ 2 Department of Orthopaedic and Trauma Surgery, University Campus Bio-Medico of Rome, 00128 Rome, \\ Italy; g.vadala@gmail.com \\ 3 Manchester Institute of Biotechnology, University of Manchester, M13 9PL Manchester, UK; \\ victoria.workman@manchester.ac.uk \\ 4 Bone Research Laboratory, Department of Oral Medicine and Periodontology, Faculty of Health Sciences, \\ School of Oral Health Sciences, University of the Witwatersrand, 2193 Johannesburg, South Africa; \\ ugo.ripamonti@wits.ac.za \\ 5 Faculty of Pharmacy and Centre for Neuroscience and Cell Biology, University of Coimbra, \\ 004-504 Coimbra, Portugal; afmendes@ff.uc.pt \\ 6 Musculoskeletal Regeneration Program, AO Research Institute Davos, Clavadelerstrasse 8, 7270 Davos, \\ Switzerland; martin.stoddart@aofoundation.org (M.J.S.); mauro.alini@aofoundation.org (M.A.) \\ * Correspondence: marta.sz@hotmail.it; Tel.: +39-095-378-2043; Fax: +39-095-378-2034
}

Received: 5 April 2016; Accepted: 6 April 2016; Published: 12 April 2016

\begin{abstract}
We are pleased to introduce the first of our Journal Club Series with the aim to review and discuss the highlights of recent papers in the field of the musculoskeletal system and associated disorders, the leitmotiv of the Journal of Functional Morphology and Kinesiology. The first edition is focused on some interesting papers published in 2015 and 2016 in the field of Articular Cartilage Tissue Engineering and Mechanical Stimulation, chosen by our Editorial Board members. We hope that this topic might tease your curiosity also in fields possibly different to your own research area, but still intrinsically connected with it. We wish you stimulating and inspiring reading.
\end{abstract}

Keywords: mechanical stimulation; tissue engineering; chondrogenesis; articular cartilage

\section{Introduction}

Articular cartilage $(\mathrm{AC})$ is a highly organized connective tissue represented by the hyaline cartilage which covers the surfaces of bones mainly in articular joints. AC is susceptible to damage, because it is aneural, avascular and highly differentiated, so its self-repair ability is limited, and once the joint is injured, this often leads to cartilage degeneration and severe disorders, such as osteoarthritis (OA). It has been reported that globally approximately 250 million people have knee OA [1], i.e., one of the most common causes for severe long-term pain and disability. To date, no efficacious treatment for OA has been reported. Thus, the development of innovative therapeutic approaches is highly sought after (desperately needed).

As a recent and a rapidly expanding field, tissue engineering may provide alternative solutions for articular cartilage repair and regeneration. For this aim, this discipline involved the use of a variety of cell types (stem cell, chondrocytes), scaffolds (biodegradable, natural or synthetic materials), 
bioactive factors (growth factors and cytokines), and physical stimuli (mechanical, electrical) to form biomimetic tissues.

It has been well-established that mechanical stimuli are crucial to the healthy development and maintenance of native articular cartilage. Consequently, mechanical stimulation became an important element in articular cartilage tissue engineering approaches [2,3].

\section{Papers Published in 2015 and 2016 Regarding the Mechanical Stimulation and Its Influence on Chondrogenesis}

\subsection{Effects of Intermittent Hydrostatic Pressure on Chondrogenic Differentiation}

\section{Highlight by Marta Anna Szychlinska}

The effect of dynamic culture and periodic compression on human mesenchymal stem cell (hMSC) proliferation and chondrogenesis has been evaluated by Guo et al. [4], which have recently developed an innovative bioreactor that can apply both shear and compressive forces to engineered tissues in dynamic culture. The latter consists of the tubular perfusion system (TPS) that creates a dynamic environment for cell culture. In this system, cells are encapsulated in 3D scaffolds and cultured in a tubular growth chamber. Nutrient and oxygen supply can be enhanced by perfusing media through the scaffolds. Contemporaneously, cells at the interface of the applied flow are exposed to shear forces. The compression force is given by the incorporation of a metal roller, which applies mechanical compression in combination with the dynamic culture conditions provided by the TPS bioreactor. In the current study, the hMSCs encapsulated into the alginate beads have been loaded into the TPS bioreactor and cultured in chondrogenic media. The study has set three objectives: (1) To investigate the hMSCs proliferation during the chondrogenesis process in the dynamic culture provided by the TPS bioreactor compared to the static culture; (2) to evaluate the effect of dynamic culture (shear force only) on hMSC chondrogenic differentiation; and (3) to evaluate if the additional cyclic compression at $0.5 \mathrm{~Hz}$ frequency would enhance the chondrogenic differentiation of hMSCs in the TPS system. From the results, based on biochemical, gene expression, histochemical and immunohistochemical analyses, it emerged that: (1) the TPS system (dynamic group) is able to increase the cell population when compared to the control (static group); (2) The static culture was preferred for differentiation compared to dynamic culture with shear force only; and (3) additional compressive force during dynamic culture can improve the chondrogenic differentiation of the hMSCs.

\subsection{Cell Surface Primary Cilia Influence on Mechanical Sensitivity of Chondroprogenitors and Hypertrophic Chondrocytes}

\section{Highlight by Gianluca Vadalà}

Deren et al. [5] hypothesized that the mechanical sensitivity of chondroprogenitors (ATDC5 cells) and hypertrophic chondrocytes is influenced by the cell surface primary cilia. To verify this hypothesis, the primary cilia have been removed from these cells by biological (transfection with intraflagellar transport protein 88 (IFT88) siRNA) and chemical (chloral hydrate) treatments. The ATDC 5 cells have then been submitted to cyclic loading at $1 \mathrm{~Hz}$ and $10 \%$ matrix deformation, in a 3D culture system (gelfoam sponges). Afterwards, the chondrogenesis marker collagen type II (Col II), hypertrophic marker collagen type X ( $\mathrm{Col} X)$, and a key regulator of chondrogenesis and hypertrophy bone morhogenic protein (BMP)-2 have been evaluated by immunohistochemical, Western Blot and RT-PCR analysis. The results suggested that primary cilia are required for mechanical activation of chondrogenesis and hypertrophy of chondroprogenitor cells, but in case of differentiated hypertrophic chondrocytes. It appears to be more limited, as complete removal of primary cilia reduced but did not eliminate totally the increase of $\mathrm{Col} \mathrm{X}$ expression in response to mechanical loading. 


\subsection{Impact of Electromagnetic Field Frequencies on Cartilage Regeneration}

\section{Highlight by Victoria Workman}

In the recent study by Yi et al. [6] the electromagnetic field impact on cartilage formation has been evaluated at different frequencies $(45$ and $7.5 \mathrm{~Hz})$ both in vitro and in vivo. For this purpose, the 3D cell-printed cartilage tissue (composed of polymeric framework and a chondrocyte-laden hydrogel) has been submitted to electromagnetic field frequencies for 14 days. After the electromagnetic field stimulation of the constructs in vitro, the latter have been implanted in the subcutaneous region of mice for 6 weeks to evaluate the effects of the pre-treatment with different electromagnetic field frequencies for promoting the cartilage formation in vivo. The results from RT-PCR, biochemical and immunofluorescence analyses showed that the frequency of $45 \mathrm{~Hz}$ enhanced the chondrogenic differentiation ability of chondrocytes (C20A4 cells), while the $7.5 \mathrm{~Hz}$ frequency suppressed this potential in vitro. Moreover, it was observed that differentially influenced chondrocyte behaviors in vitro pre-treatment with different electromagnetic field frequencies influenced different capabilities for the enhancement of cartilage regeneration in vivo without additional electromagnetic field treatment.

\subsection{Effect of Dynamic Culture and Periodic Compression on Human Mesenchymal Stem Cell Chondrogenesis}

\section{Highlight by Ugo Ripamonti}

The aim of this interesting study by Li et al. [7] was to evaluate the effects of intermittent hydrostatic pressure (IHP) on the chondrogenic differentiation of cartilage progenitor cells cultured in alginate beads. The cartilage progenitor cells used for the study and included in alginate beads were isolated from the knee joint cartilage of rabbits and infrapatellar fat pad-derived stem cells (FPSCs), and chondrocytes were used as a control. The IHP was imposed at $5 \mathrm{Mpa}$ and $0.5 \mathrm{~Hz}$ for $4 \mathrm{~h}$ /day for 1, 2, or 4 weeks. The analysis performed (scratch and live/dead assays, histological and immunohistochemical analysis, and RT-PCR) revealed that the IHP enhanced the chondrogenic differentiation capacity of the cells and that it was stronger in cartilage progenitor cells when compared to FPSCs and controls. Important studies are now casting serious doubts on the biological outcome of transplanting chondrogenic precursor and/or stem cells to regenerate AC in severe arthritis conditions. Recently, Mao's group at the Tissue Engineering and Regenerative Medicine Laboratory, NY, USA have shown regeneration of the articular cartilage surface of the rabbit synovial joint by cell homing of locally activated and re-deployed progenitor cells. Instead of transplanting cells, the proof of concept approach was to implant TGF- $\beta_{3}$-infused scaffolds that would stimulate endogenous cells to differentiate into mature chondrocytes [8]. My own experience using the hTGF- $\beta_{3}$ isoform in the non-human primate Papio ursinus, has also indicated that the soluble molecular signal is powerful enough to rapidly differentiate a variety of progenitor cells into the osteoblastic phenotype, and to induce the expression of a variety of profiled bone morphogenetic proteins genes that set into motion the rapid ripple-like cascade of bone differentiation by induction $[9,10]$.

\subsection{The Effect of Mechanical Stimulation and Adenoviral-Mediated Over-Expression of BMP-2 on the Chondrogenesis of Human Articular Cartilage Progenitor Cells}

\section{Highlight by Alexandrina Ferreira Mendes}

Articular cartilage progenitor cells (ACPCs) represent a potential alternative cell source for articular cartilage repair approaches, and have been shown to be resistant to hypertrophy. A recent and interesting study by Neumann et al. [11] investigated the effect of mechanical stimulation and BMP-2 overexpression, alone and in combination, on the chondrogenic differentiation capacity of human ACPCs (hACPCs). The cells were seeded in fibrin-polyurethane composite scaffolds and cultured in a defined chondro-permissive medium lacking exogenous growth factors. The cells in 
the composite scaffolds were successfully transduced with an adenovirus encoding human BMP-2. Transduced and non-transduced three dimensional hACPCs constructs were cultured for 7 or 28 days under static (unloaded) conditions or with the application of a complex mechanical stimulation protocol (dynamic compression at $1 \mathrm{~Hz}, 0.4-0.8 \mathrm{~mm}$, and shear stress at $1 \mathrm{~Hz}, \pm 25^{\circ}$, superimposed on a static offset strain of $0.4 \mathrm{~mm}$ ) previously shown to induce the chondrogenesis of human bone marrow mesenchymal stem cells, using a custom built bioreactor. The biochemical, gene expression, histological and immunohistochemical analyses showed that the loading protocol applied is sufficient to initiate chondrogenesis, demonstrating that hACPCs are mechanoresponsive and that appropriate mechanical stimulation is an effective way of promoting the chondrogenic differentiation of these cells. Furthermore, over-expression of BMP-2, independently of mechanical stimulation, also increased the expression of some chondrogenic markers (aggrecan, collagen II), but unlike mechanical stimulation, gene expression levels of hypertropic markers (collagen $\mathrm{X}$ and alkaline phosphatase) increased simultaneously. In summary, this study highlights the feasibility of using appropriate mechanical stimulation protocols to promote the chondrogenesis of hACPCs for cartilage tissue engineering. Nevertheless, further studies are required to assess the long term properties and phenotypic stability of the engineered cartilage produced under such circumstances.

\subsection{Ageing and Reduction of Mechanically-Induced Smad2/3P Signaling Activation in Articular Cartilage}

\section{Highlight by Martin J. Stoddart and Mauro Alini}

Frequently, studies into mechanoregulation of chondrocytes and cartilage is performed using young healthy cartilage. However, it is elderly cartilage where degeneration and potential dysregulation occurs, and it is in these elderly patients where therapies are most frequently required. In the study by Madej et al. [12] they compared the response of young (1-3 years) and old (10-13 years) bovine cartilage to $1 \mathrm{~Hz}$ cyclical compression at either 3 or $12 \mathrm{MPa}$. In young cartilage, mechanically induced upregulation of bone morphogenetic protein 2 (BMP2), connective tissue growth factor and, at higher compression, TGF- $\beta_{1}$ was observed. These changes were not seen in elderly cartilage. More strikingly the activation of Smad2/3P signaling seen in young cartilage was completely absent in elderly cartilage. As the Smad2/3P pathway is known to be protective of articular cartilage, this link with age, mechanobiology and signal pathways offers exciting possibilities for further age related diseases such as osteoarthritis.

Acknowledgments: A special thanks goes to the Editorial Board members who committed to this new initiative of the Journal with much enthusiasm and interest.

Author Contributions: All authors contributed equally to this work, each author wrote the highlight indicated, each author reviewed the proofs before publication.

Conflicts of Interest: The authors declare no conflict of interest.

\section{Abbreviations}

The following abbreviations are used in this manuscript:

AC

ACPCs

BMP

FPSCs

Hmsc

IFT88

IHP

OA

TPS
Articular Cartilage

Articular Cartilage Progenitor Cells

Bone Morphogenic Protein

Fat Pad-Derived Stem Cells

Human mesenchymal stem cell

Intraflagellar Transport Protein 88

Intermittent Hydrostatic Pressure

Osteoarthritis

Tubular Perfusion System 


\section{References}

1. Vos, T.; Flaxman, A.D.; Naghavi, M.; Lozano, R.; Michaud, C.; Ezzati, M.; Shibuya, K.; Salomon, J.A.; Abdalla, S.; Aboyans, V.; et al. Years lived with disability (YLDs) for 1160 sequelae of 289 diseases and injuries 1990-2010: A systematic analysis for the Global Burden of Disease Study 2010. Lancet 2012, 380, 2163-2196. [CrossRef]

2. Grad, S.; Eglin, D.; Alini, M.; Stoddart, M.J. Physical stimulation of chondrogenic cells in vitro: A review. Clin. Orthop. Relat. Res. 2011, 469, 2764-2772. [CrossRef] [PubMed]

3. Schätti, O.; Grad, S.; Goldhahn, J.; Salzmann, G.; Li, Z.; Alini, M.; Stoddart, M.J. A combination of shear and dynamic compression leads to mechanically induced chondrogenesis of human mesenchymal stem cells. Eur. Cell. Mater. 2011, 22, 214-225. [PubMed]

4. Guo, T.; Yu, L.; Lim, C.G.; Goodley, A.S.; Xiao, X.; Placone, J.K.; Ferlin, K.M.; Nguyen, B.B.; Hsieh, A.H.; Fisher, J.P. Effect of dynamic culture and periodic compression on human mesenchymal stem cell proliferation and chondrogenesis. Ann. Biomed. Eng. 2015. [CrossRef] [PubMed]

5. Deren, M.E.; Yang, X.; Guan, Y.; Chen, Q. Biological and chemical removal of primary cilia affects mechanical activation of chondrogenesis markers in chondroprogenitors and hypertrophic chondrocytes. Int. J. Mol. Sci. 2016, 17. [CrossRef] [PubMed]

6. Yi, H.G.; Kang, K.S.; Hong, J.M.; Jang, J.; Park, M.N.; Jeong, Y.H.; Cho, D.W. Effects of electromagnetic field frequencies on chondrocytes in 3D cell-printed composite constructs. J. Biomed. Mater. Res. 2016. [CrossRef] [PubMed]

7. Li, Y.; Zhou, J.; Yang, X.; Jiang, Y.; Gui, J. Intermittent hydrostatic pressure maintains and enhances the chondrogenic differentiation of cartilage progenitor cells cultivated in alginate beads. Dev. Growth. Differ. 2016, 58, 180-193. [CrossRef] [PubMed]

8. Lee, C.H.; Cook, J.L.; Mendelson, A.; Moioli, E.K.; Yao, H.; Mao, J.J. Regeneration of the articular surface of the rabbit synovial joint by cell homing: A proof of concept study. Lancet 2010, 376, 440-448. [CrossRef]

9. Ripamonti, U.; Duarte, R.; Ferretti, C. Re-evaluating the induction of bone formation in primates. Biomaterials 2014, 35, 9407-9422. [CrossRef] [PubMed]

10. Ripamonti, U.; Dix-Peek, T.; Parak, R.; Milner, B.; Duarte, R. Profiling bone morphogenetic proteins and transforming growth factor- $\beta_{\mathrm{S}}$ by hTGF- $\beta_{3}$ pre-treated coral-derived macroporous constructs: The power of one. Biomaterials 2015, 49, 90-102. [CrossRef] [PubMed]

11. Neumann, A.J.; Gardner, O.F.; Williams, R.; Alini, M.; Archer, C.W.; Stoddart, M.J. Human articular cartilage progenitor cells are responsive to mechanical stimulation and adenoviral-mediated overexpression of bone-morphogenetic protein 2. PLoS ONE 2015, 10, e0136229.

12. Madej, W.; van Caam, A.; Blaney Davidson, E.N.; Hannink, G.; Buma, P.; van der Kraan, P.M. Ageing is associated with reduction of mechanically-induced activation of Smad2/3P signaling in articular cartilage. Osteoarthr. Cartil. 2016, 24, 146-157. [CrossRef] [PubMed]

(C) 2016 by the authors; licensee MDPI, Basel, Switzerland. This article is an open access article distributed under the terms and conditions of the Creative Commons Attribution (CC-BY) license (http://creativecommons.org/licenses/by/4.0/). 